\title{
PASSer: Prediction of Allosteric Sites Server
}

\author{
Hao Tian, ${ }^{\dagger}$ Xi Jiang, ${ }^{\ddagger}$ and Peng Tao ${ }^{*, \dagger}$ \\ $\dagger$ Department of Chemistry, Center for Research Computing, Center for Drug Discovery, \\ Design, and Delivery (CD4), Southern Methodist University, Dallas, Texas, United States \\ of America \\ $\ddagger$ Department of Statistical Science, Southern Methodist University, Dallas, Texas, United \\ States of America \\ E-mail: ptao@smu.edu
}

\begin{abstract}
Allostery is considered important in regulating protein's activity. Drug development depends on the understanding of allosteric mechanisms, especially the identification of allosteric sites, which is prerequisite in drug discovery and design. Many computational methods have been developed for allosteric site prediction using pocket features and dynamics information. Here, we provide a novel ensembled model, consisting of eXtreme gradient boosting (XGBoost) and graph convolutional neural network (GCNN) to predict allosteric sites. Our model can learn both physical properties and topology structure without any prior information and exhibited good performance under several indicators. Prediction results have shown that $84.9 \%$ of allosteric pockets in the testing proteins appeared in the top 3 positions. The PASSer: Protein Allosteric Sites Server (https://passer.smu.edu), along with a command line interface (CLI, https://github.com/smutaogroup/passerCLI) provide insights for further analysis in drug discovery.
\end{abstract}




\section{Introduction}

Allostery is the process that proteins transmit the perturbation caused by the effect of binding at one site (active site) to another site (allosteric site). ${ }^{1}$ The allosteric process is fundamental in regulation of activity. Compared with non-allosteric drugs, allosteric drugs have many benefits: they are conserved and highly specific; ${ }^{2}$ they can either activate or inhibit proteins; they can be used together with orthosteric (non-allosteric) drugs. While allosteric drugs are important in pharmaceutical industry, ${ }^{3}$ they are poorly discovered ${ }^{4}$ due to the reason that most allosteric mechanisms remain unclear ${ }^{5}$ and the identification of allosteric sites is prerequisite for further study.

Many allosteric site prediction methods have been developed using molecular dynamics (MD), ${ }^{6}$ normal mode analysis, ${ }^{7}$ two-state $\mathrm{G} \bar{o}$ models $^{8}$ and machine learning (ML) models. ${ }^{9-11}$ Among all the existing methods, AllositePro, ${ }^{12}$ AlloPred, ${ }^{13}$ SPACER ${ }^{14}$ and PARS ${ }^{15}$ are available as web servers or open-source packages. These previous studies have shown that it is promising to identify allosteric sites by combining static pocket features with protein dynamics features. The static features are calculated by site descriptors describing physical properties of allosteric pockets while the dynamics features are extracted from MD simulations or purturbations.

Machine learning methods have shown to be superior in the classification of protein pockets. For example, Allosite ${ }^{11}$ and AlloPred ${ }^{13}$ used support vector machine (SVM) ${ }^{16}$ with optimized features while Chen et al. ${ }^{17}$ used random forest $(\mathrm{RF})^{18}$ to build a threeway predictive model. With the development of ML, more advanced models have been

developed and can contribute to the allosteric site classification. eXtreme gradient boosting ${ }^{19}$ (XGBoost) is one of the most powerful machine learning techniques in classification. It is an implementation of gradient boosting algorithm with regularized term to reduce overfitting. Compared with SVM and RF, XGBoost achieved higher prediction results from proteinprotein interactions ${ }^{20}$ to hot spots. ${ }^{21}$

While physical properties are mainly covered in many methods, the topology information 
is largely ignored and is considered important in classifying pockets. In order to prob the geometry feature, an atomic graph is constructed for each pocket. Atoms were treated as nodes and the pairwised bond distances were calculated as edges. ${ }^{9}$ Graph convolutional neural network (GCNN), ${ }^{22}$ a popular concept in deep learning, has been applied in biologicalrelated predictions, ranging from chemical reactions, ${ }^{23}$ molecular properties ${ }^{24}$ to drug-target interactions ${ }^{25}$.

In this study, protein pockets are predicted using an ensembled model consisting of XGBoost and GCNN. This model can learn both physical properties and topology information of allosteric pockets and is demonstrated to be superior than single XGBoost and GCNN model. Various performance indicators validated the success of this ensembled model compared with previous methods.

\section{Methods}

\subsection{Protein Database}

The data used in the current work was collected from Allosteric Database (ASD) ${ }^{26}$. There are in total of 1946 entries of allosteric site information with different proteins and modulators. To ensure data quality, 90 proteins were selected using previous rules ${ }^{11}$ : protein structures with either resolution below $3 \AA$ or missing residues in the allosteric sites were removed; redundant proteins in the rest dataset that have over $30 \%$ sequence identity were filtered out.

\section{$2.2 \quad$ Site Descriptors}

FPocket algorithm ${ }^{27}$ was applied to detect pockets from the surface of the selected proteins. Each pocket was labeled as either one (positive) if it contains at least one resiude or zero (negative). Therefore, one protein structure might have more than one positive label. A total of 2246 pockets were detected with 119 pockets being labeled as allosteric sites. There 
Table 1: Site Descriptors Calculated by FPocket

\begin{tabular}{cc}
\hline Number & Description \\
\hline 1 & Score \\
2 & Druggability score \\
3 & Number of alpha spheres \\
4 & Total solvent-accessible surface area (SASA) \\
5 & Polar SASA \\
6 & Apolar SASA \\
7 & Volume \\
8 & Mean local hydrophobic density \\
9 & Mean alpha sphere radius \\
10 & Mean alpha sphere solvent access \\
11 & Apolar alpha sphere proportion \\
12 & Hydrophobicity score \\
13 & Volume score \\
14 & Polarity score \\
15 & Charge scores \\
16 & Proportion of polar atoms \\
17 & Alpha sphere density \\
18 & Centre of mass - alpha sphere max distance \\
19 & Flexibility \\
\hline
\end{tabular}

are 19 features calculated by FPocket as shown in Table 1.

\subsection{Pearson Correlation Coefficient}

Pearson correlation coefficient (PCC) measures the linear correlation of two variables. Given a pair of variables $X$ and $Y$ as $\left\{\left(x_{1}, y_{1}\right), \ldots,\left(x_{n}, y_{n}\right)\right\}$, PCC $\left(r_{X, Y}\right)$ is calcualted as

$$
r_{X, Y}=\frac{\sum_{i=1}^{n}\left(x_{i}-\bar{x}\right)\left(y_{i}-\bar{y}\right)}{\sqrt{\sum_{i=1}^{n}\left(x_{i}-\bar{x}\right)^{2}} \sum_{i=1}^{n}\left(y_{i}-\bar{y}\right)^{2}}
$$

where $n$ is the sample size and $\bar{x}, \bar{y}$ are sample means. PCC has a value between -1 and +1 . The absolute value indicates the degree of correlation with +1 and -1 represent positive and negative correlations, respectively. 


\section{4 eXtreme Gradient Boosting}

Extreme gradient boosting ${ }^{19}$ is an ensemble learning method that combines several decision trees in sequence.

Let $D=\left\{\left(x_{i}, y_{i}\right)\left(|D|=n, x_{i} \in R^{m}, y_{i} \in R^{n}\right)\right\}$ represents a dataset with $m$ features and $n$ labels. The $j$-th decision tree in XGBoost predicts a sample $\left(x_{i}, y_{i}\right)$ by:

$$
g_{j}\left(x_{i}\right)=w_{q}\left(x_{i}\right)
$$

where $w_{q}$ is the leaf weights of this decision tree. The final prediction of XGBoost is given by the summation of predictions from each decision tree:

$$
\hat{y}_{i}=\sum_{j=1}^{M} g_{j}\left(x_{i}\right)
$$

where $M$ is the total number of decision trees. To overcome overfitting introduced by decision trees, the objective function in XGBoost is composed of a loss function $l$ and a regularization term $\Omega$ :

$$
\operatorname{obj}(\theta)=\sum_{i=1}^{N} l\left(y_{i}, \hat{y}_{i}\right)+\sum_{j=1}^{M} \Omega\left(f_{i}\right)
$$

where $\Omega(f)=\gamma T+\frac{\lambda}{2} \sum_{l=1}^{T} \omega_{l}^{2}$ with $T$ represents the number of leaves and $\gamma, \lambda$ are regularization parameters.

During training, XGBoost iteratively adds new decision tree. The prediction of the $t$-th iteration is expressed as:

$$
\hat{y}_{i}{ }^{(t)}=\hat{y}_{i}{ }^{(t-1)}+g_{t}\left(x_{i}\right)
$$

Correspondingly, the objective function of the $t$-th iteration is: 


$$
\mathrm{obj}^{(t)}=\sum_{i=1}^{N} l\left(y_{i}, \hat{y}_{i}^{(t-1)}+g_{t}\left(x_{i}\right)\right)+\Omega\left(f_{i}\right)
$$

XGBoost introduces both first derivative and second derivative of the loss function. By applying Taylor expansion on the objective function at second-order, the objective function of the $t$-th iteration can be expressed as:

$$
\begin{aligned}
\operatorname{obj}^{(t)} & \simeq \sum_{i=1}^{N}\left[l\left(y_{i}, \hat{y}_{i}^{(t-1)}\right)+\partial_{\hat{y}^{(t-1)}} l\left(y_{i}, \hat{y}^{(t-1)}\right) f_{t}\left(x_{i}\right)\right. \\
& \left.+\frac{1}{2} \partial_{\hat{y}^{(t-1)}}^{2} l\left(y_{i}, \hat{y}^{(t-1)}\right) f_{t}^{2}\left(x_{i}\right)\right]+\Omega\left(f_{i}\right)
\end{aligned}
$$

XGBoost can not only predict the label of sample data but also the corresponding probability. For one pocket, XGBoost outputs the probability of this pocket being allosteric pocket. This pocket is labled as positive (allosteric) if the probability is over $50 \%$ or negative otherwise.

There are only $5.3 \%$ of positive labels in this binary classification job, indicating the input dataset is highly imbalanced. To focus more on the limited positive labels, XGBoost uses "scale_pos_weight", a parameter to control the balance of positive and negative weights,

during training. A typical value of this weight equals to the sum of negative samples versus the sum of positive samples.

In the current study, the values of the maximum tree depth for base learners and the weight for positive labels were fine tuned while other parameters used default values. The XGBoost algorithm is implemented using Scikit-learn package ${ }^{28}$ version 0.23.2.

\subsection{Graph Convolutional Neural Network}

Graph convolutional neural network in this work follows this formula ${ }^{22}$ :

$$
H^{(l+1)}=\operatorname{ReLU}\left(\tilde{D}^{-\frac{1}{2}} \tilde{A} \tilde{D}^{-\frac{1}{2}} H^{(l)} W^{(l)}\right)
$$




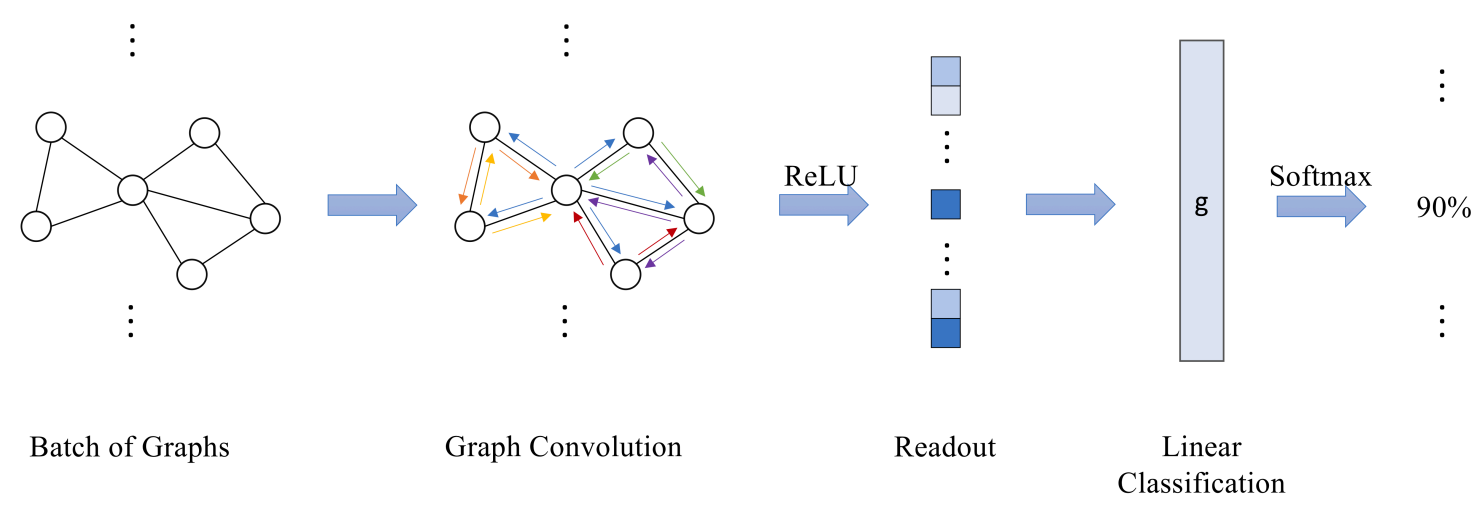

Figure 1: 1-layer graph convolutional neural network architecture. 1-layer GCNN is composed of an input layer, a graph convolution layer, a readout layer and a linear classification layer. For an atomic graph of a pocket, GCNN predicts the probability of this pocket being an allosteric pocket.

where $H^{(l)}$ and $H^{(l+1)}$ represents the $l^{\text {th }}$ and $l+1^{\text {th }}$ layer, respectively. $H^{(l)} \in \mathbb{R}^{N \times D}$, where $N$ is the number of nodes and $D$ is the number of features. Rectified linear unit $(\operatorname{ReLU}(x)=\max (0, x))$ is used as the activation function. $W^{(l)}$ denotes the weight matrix in the $l^{\text {th }}$ layer. $D$ and $A$ represent degree matrix and adjacent matrix, respectively, with $\tilde{D}_{i i}=\sum_{j} \tilde{A}_{i j}$. Renormalization trick ( symbol) is applied for the undirected graph $G$ where each node is added with a self-connection. Therefore, $\tilde{A}=A+I_{N}$ where $I_{N}$ is the identity matrix.

A graph readout is calculated through the average of node features for each graph.

$$
h_{g}=\frac{1}{|\mathcal{V}|} \sum_{v \in \mathcal{V}} h_{v}
$$

where $h_{g}$ is the readout result of graph $g$ and $h_{v}$ is the node feature in node $v . \mathcal{V}$ represents all nodes in graph $g$.

An example of 1-layer GCNN model is shown in Figure 1. A graph is first fed into a convolution layer. In-degree of each node was calculated as the node feature. Since the atomic graph is undirected, the in-degree equals to the out-degree in each node. Graph feature was calculated as the average of node features in the readout layer with ReLU activation function. The output was further fed into a linear classification layer $g$, which predicts the probability 
Table 2: Binary Classification Results

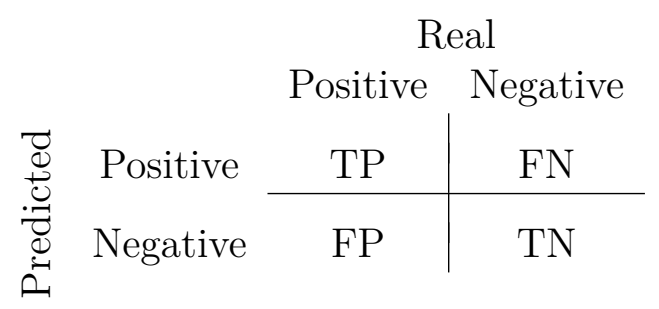

of being an allosteric pocket. Previous study ${ }^{29}$ showed the limitation of 1-layer GCNN and thus a 2-layer GCNN model (two graph convolution layers) was applied.

In order to overcome the potential failure in traning GCNN with imbalanced dataset, the ratio between negative labels and positive labels was finetuned. Specifically, in each protein, allosteric pockets (positive samples) were used in full while non-allosteric pockets (negative samples) were used in partial. Each negative sample was randomly selected and the total number of non-allosteric pockets equals the number of allosteric pockets times the ratio value.

In constructing atomic graphs, the threshold of bond distance was also finetuned. Each atom was considered as a node in the atomic graph and the pairwised distances between atoms were calculated. If the distance is below a speficied threshold, an edge is constructed connecting the two related atoms. Therefore, the distance threshold controls the degree of local connectivity.

The GCNN model is implemented using Deep graph library (DGL) package ${ }^{30}$ version 0.4.3.

\subsection{Performance Indicators}

For binary classification, the results can be classified as Table 2. TP, FN, FP and TN represent true positive, false negative, false positive and true negative, respectively.

Various indicators were used to quantify model performace: precision (TP / (TP + FP)) measures how good the model can predict real positive labels; accuracy $((\mathrm{TP}+\mathrm{TN}) /(\mathrm{TP}+$ $\mathrm{FP}+\mathrm{FN}+\mathrm{TN})$ ) measures the overall classification accuracy; recall (or named as sensitivity, 
$\mathrm{TP} /(\mathrm{TP}+\mathrm{FN}))$ and specifity $(\mathrm{TN} /(\mathrm{TN}+\mathrm{FP}))$ together measures the ability to classify $\mathrm{TP}$ and TN; F1 score $(2 *$ precision * recall / (precision + recall $))$ is the weighted average of precision and recall. The higher the values of these indicators, the better the performance of model.

A receiver operating characteristic (ROC) curve was applied as another indicator to test model performace in binary classification. ROC curve is plotted as TP rate against FP rate with different threshold settings. The area under curve (AUC) is calculated for quantification. A well-behaved model is expected to have a AUC value of 1.0 while a dummy model should reach a AUC value of 0.5 .

The predicted ranking of allosteric pockets was also considered as an important indicator. Specifically, for every allosteric pocket, the predicted ranking (predicted probability of being an allosteric site in descending order) was recorded and classified as first, second, third or other positions. In other words, if a pocket is an allosteric pocket, the ranking result indicates how probable this pocket is predicted top among all pockets in the same protein. A model with good performace should rank an allosteric pocket in the top positions.

\section{Results}

\subsection{Feature Exploration}

The distribution of 19 features is shown in Figure 2. While some exhibited long tail distributions such as feature 1 (Score) and 2 (Druggability score), data normalization is not necessary since XGBoost does not require normal distribution. Instead, XGBoost, as well as other tree based models, only focuses on the order and whether features are normalized or not does not affect the final predictions. To better distinguish the feature distribution for allosteric sites and non-allosteric sites, violin plots are displayed in Figure 3.

The correlation matrix between features is shown in Figure 4. Several features exhibited high correlations, for example, feature 3 (Number of alpha spheres), 4 (Total SASA), 5 (Polar 

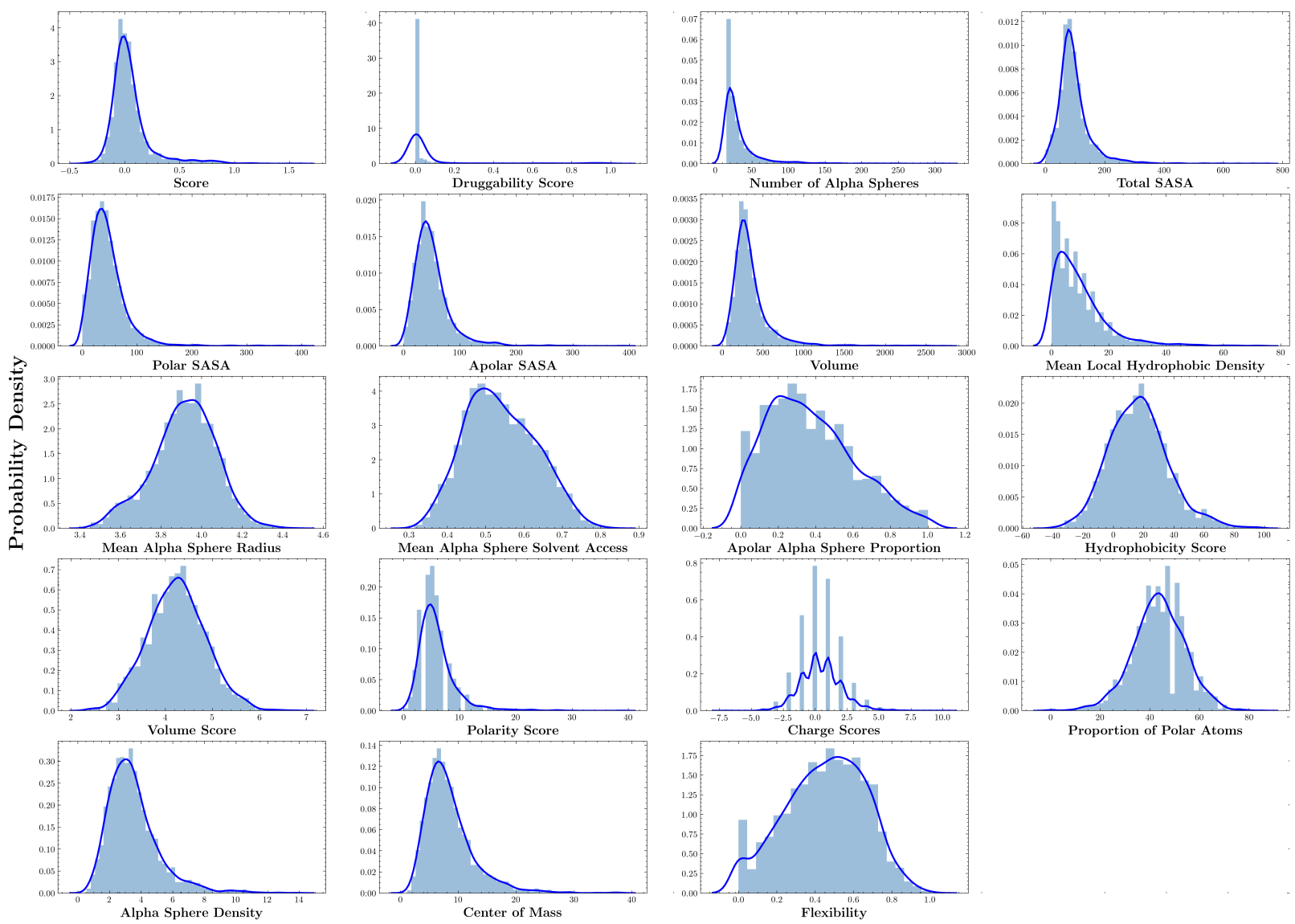

Figure 2: Distribution of features. A histogram plot and a kernel density estimate (blue line) is shown for each feature.

SASA), 6 (Apolar SASA) and 7 (Volume) are highly correlated with each other. Feature 17 (Alpha sphere density) and 18 (Centre of mass) were also strong correlated with these five features.

For each feature value in a pocket, the inner ranking refers to the ranking position of this feature among all values in the same protein in descending order. Similar with the definition of inner ranking, the overall ranking refers to the ranking position of this feature among all values in the overall dataset. Both ranking feature sets were normalized. The correlation between original features and these two ranking feature sets were calculated and shown in Figure 3. The great negative values indicate strong negative correlation between original features and ranking features. While feature rankings were calculated and applied as additional features in a previous study, ${ }^{13}$ in the current dataset, the high correlation 

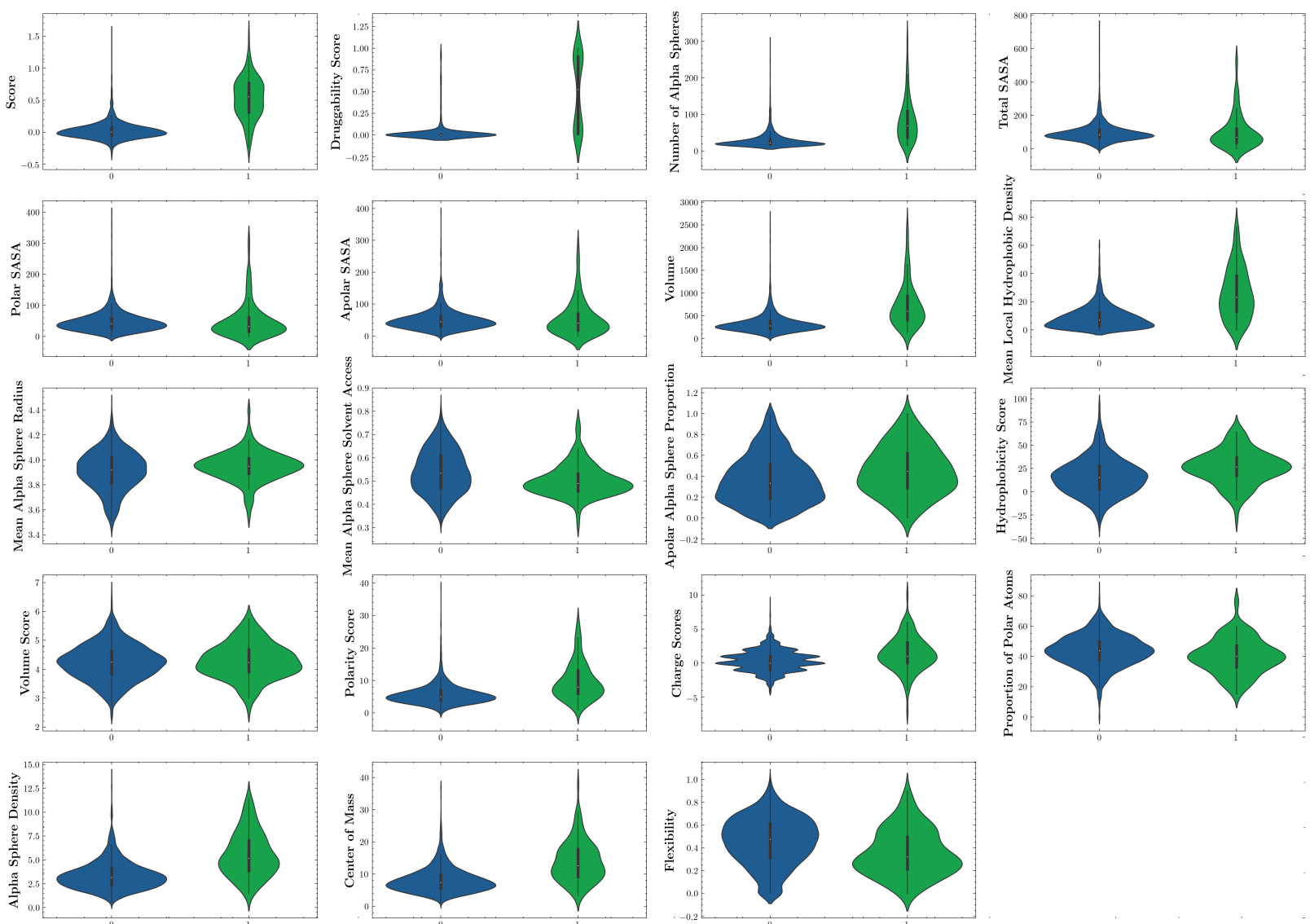

Class

Figure 3: Violin plot of features. The distribution of allosteric pockets (class 1) and non-allosteric pockets (class 0 ) with probability density is plotted for each feature.

indicates that the ranking features can provide little information and thus were discarded.

\subsection{Prediction performance of XGBoost}

XGBoost model can overcome the problem of data imbalance by controlling the weight between negative labels and positive labels. This parameter was finetuned along with the maximum depth of trees. The results are shown in Figure 5. Two sets of parameters reached high F1 score and both were selected in the final model. Therefore, the final XGBoost model is composed of two models, each with one set of parameters. The results of any given pocket is the averaged results predicted by these two models.

The results of finetuned XGBoost model are shown in Table 4. Compared with the reference results, XGBoost model exihibited higher accuracy, precision, specifity and ROC 


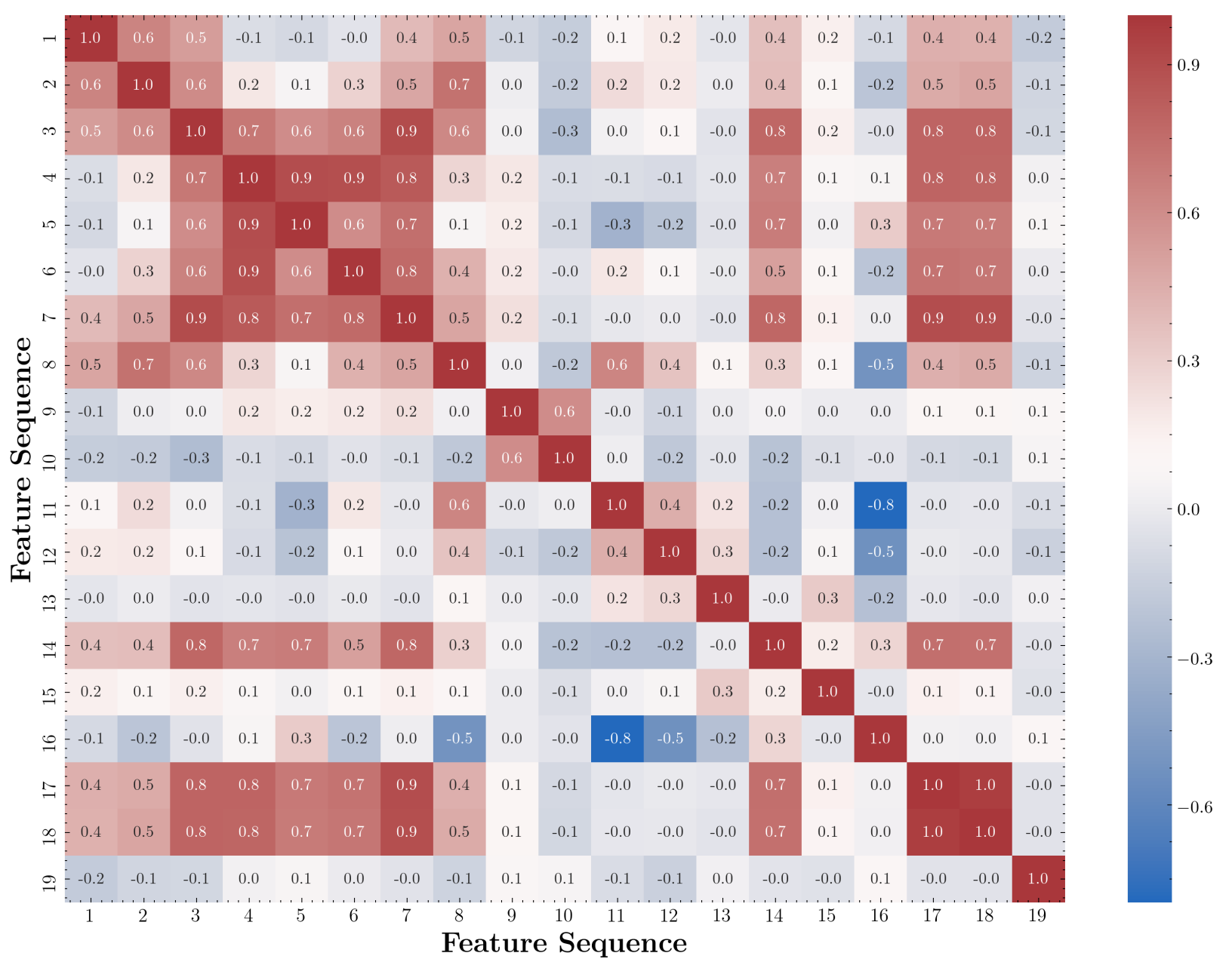

Figure 4: Heatmap of correlation matrix between features. The numbers in feature sequence correspond to the numbers in Table 1. The correlation matrix is symmetrical along the diagonal. White color represents no correlation between the two related features while red and blue represent positive and negative correlations, respectively. The darker the color, the greater the correlation.

AUC value with comparable results in recall and F1 score. Therefore, XGBoost model shows good performance in allosteric site prediction.

\subsection{Prediction performancec of GCNN}

Different with XGBoost model, GCNN fails to deal with highly imbalanced dataset. In order to deal with this problem, the ratio between negative labels and positive labels was evaluated first. The results are shown in Figure 6A. Ratio of 2 (number of negative labels : number of positive labels $=2: 1$ ) was selected. The distance threshold was further 
finetuned and the resuls are shown in Figure $6 \mathrm{~B} .10 \AA$ was selected as the distance cutoff when constructing atomic graphs.

The results of finetuned GCNN model in 10 independent runs are shown in Table 4. It is shown that, compared with XGBoost, GCNN is less powerful in classifying allosteric sites. However, it is expected that combining XGBoost and GCNN should have better performance compared with either model.

\subsection{Prediction performancec of Ensembled Model}

The ensembled model is composed of XGBoost model and GCNN model. For a given pocket, physical properties and ranking information was calculated and fed into the trained XGBoost model; atomic graph was fed into the trained GCNN model. A final probability is calculated as the averaged probability of the two models. This final model is believed to learn both physical properties and topology features of protein pockets. The combined

Table 3: Correlation Between Original Features and Additional Rankings

\begin{tabular}{ccc}
\hline Feature Number & Inner Ranking & Overall Ranking \\
\hline 1 & -0.7 & -0.8 \\
2 & -0.5 & -0.5 \\
3 & -0.6 & -0.7 \\
4 & -0.8 & -0.8 \\
5 & -0.8 & -0.8 \\
6 & -0.8 & -0.8 \\
7 & -0.7 & -0.8 \\
8 & -0.8 & -0.9 \\
9 & -0.9 & -1.0 \\
10 & -0.9 & -1.0 \\
11 & -0.9 & -1.0 \\
12 & -0.9 & -1.0 \\
13 & -0.9 & -1.0 \\
14 & -0.8 & -0.8 \\
15 & -0.9 & -0.9 \\
16 & -0.9 & -1.0 \\
17 & -0.9 & -0.9 \\
18 & -0.9 & -0.9 \\
19 & -0.6 & -1.0 \\
\hline
\end{tabular}




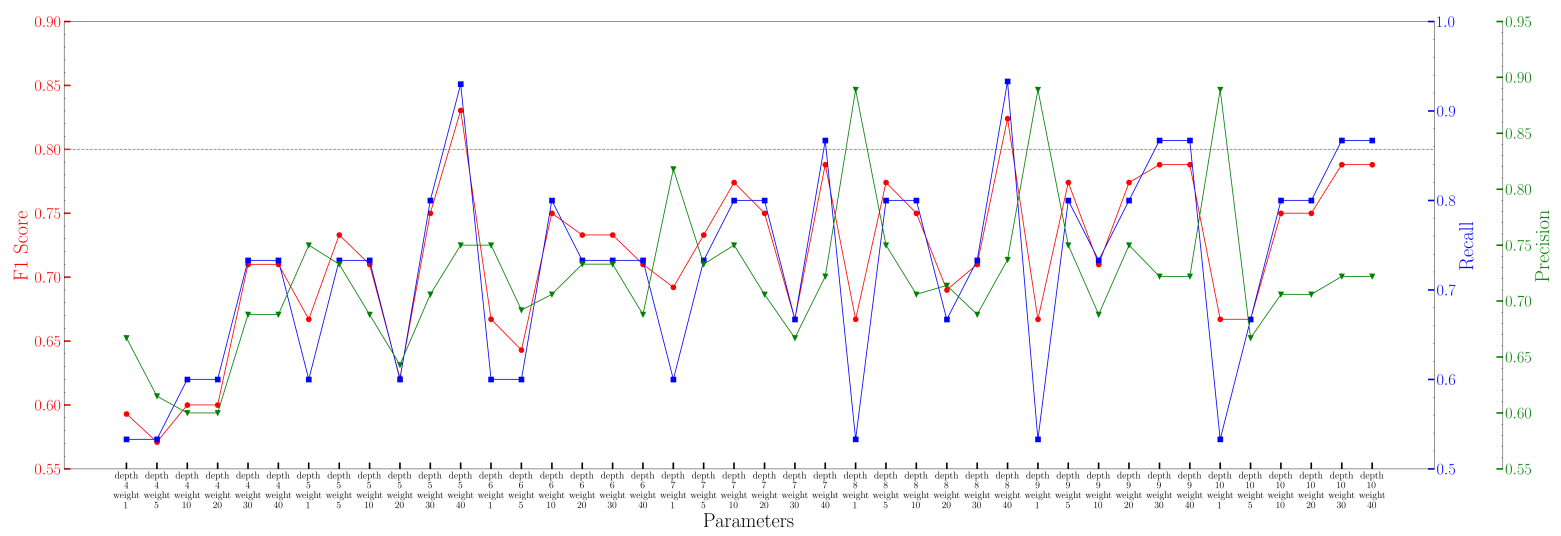

Figure 5: Finetune results of XGBoost model. Both weight and depth were finetuned. Two sets of parameters, depth 5 weight 40 and depth 8 weight 40, exhibited exceptional high F1 score and were selected.

Table 4: Performance of Different Models

\begin{tabular}{ccccccc}
\hline & Accuracy & Recall & Precision & Specifity & F1 score & ROC AUC \\
\hline \multirow{2}{*}{ XGBoost } & 0.969 & 0.799 & 0.732 & 0.982 & 0.764 & 0.897 \\
& $\pm 0.002^{a}$ & \pm 0.023 & \pm 0.030 & \pm 0.003 & \pm 0.016 & \pm 0.016 \\
GCNN & 0.923 & 0.604 & 0.427 & 0.943 & 0.500 & 0.832 \\
& \pm 0.006 & \pm 0.023 & \pm 0.046 & \pm 0.007 & \pm 0.031 & \pm 0.015 \\
Ensembled model & 0.974 & 0.847 & 0.726 & 0.980 & 0.782 & 0.914 \\
& \pm 0.010 & \pm 0.095 & \pm 0.085 & \pm 0.013 & \pm 0.072 & \pm 0.018 \\
Allosite $^{11}$ & 0.962 & 0.852 & 0.688 & 0.970 & 0.761 & 0.911 \\
\hline \multicolumn{5}{c}{ Standard error (SE) } & Standard deviation $(\mathrm{SD}) / \sqrt{\text { sample size }}$ &
\end{tabular}

results are shown in Table 4. Compared with the XGBoost model, the ensembled model had $6.00 \%$ increase in recall with $0.82 \%$ decrease in precision, together leading to $2.89 \%$ increase in F1 score. The AUC ROC value also had $1.89 \%$ increase.

The predicted ranking of allosteric pockets was calculated for the top 3 positions. $60.7 \%$ of allosteric pockets appear in the first position, while $81.6 \%$ in the top 2 and $84.9 \%$ in the top 3. In other words, if a pocket is an allosteric pocket, there is a probability of $84.9 \%$ that it can be predicted as top 3 among all detected pockets in the same protein. Our results outperformed the previous results as shown in Table 5. However, it should be noted that the type and number of testing proteins are different. 
(A)

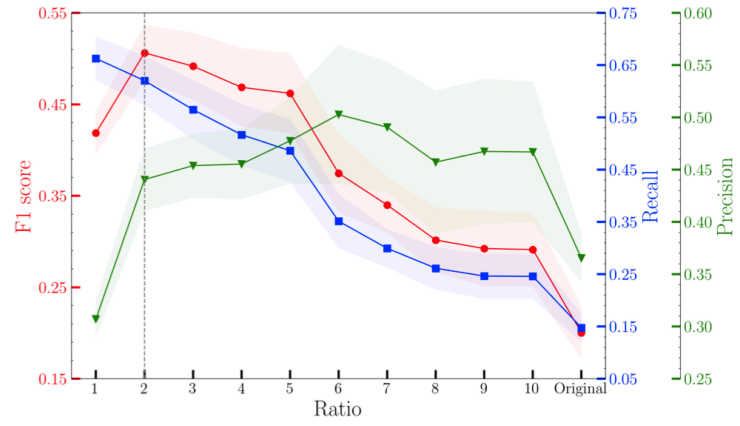

(B)

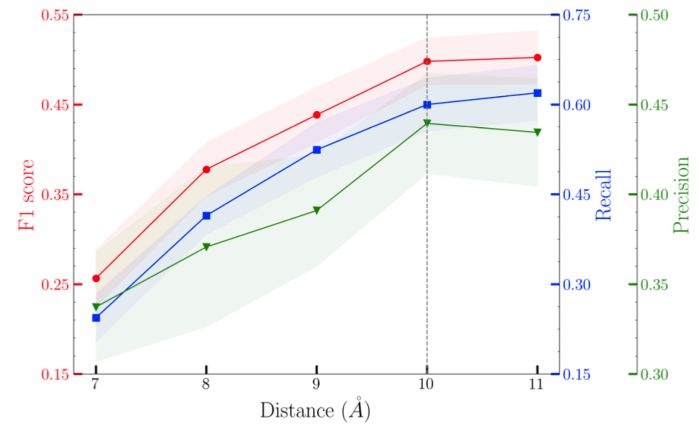

Figure 6: Finetune results of GCNN model. (A) Results for the ratio between number of negative labels and number of positive labels. Each ratio was run 10 times independently. Ratio of 2 was considered reaching a balance between recall and precision. (B) Results for the distance threshold. Each threshold was run 10 times independently.

Table 5: Probabilities of Ranking An Allosteric Pocket in Top 3

\begin{tabular}{cccc}
\hline & Top 1 & Top 2 & Top 3 \\
\hline PARS $^{15}$ & $44 \%$ & $62 \%$ & $73 \%$ \\
AlloPred $^{13}$ & $57.5 \%$ & $70.0 \%$ & $\mathrm{NA}^{a}$ \\
Ensembled Model & $60.7 \%$ & $81.6 \%$ & $84.9 \%$ \\
\hline${ }^{a}$ Not available in the reported results.
\end{tabular}

\subsection{Novel Allosteric Sites Prediction}

To test the stability of this ensembled model, proteins that are not in the ASD were predicted. The prediction results are shown in Figure 7. Two different types of allosteric proteins were used as examples. The second PDZ domain (PDZ2) is a dynamics-driven protein in human PTP1E protein that experiences allosteric process upon binding with peptide. ${ }^{31}$ The light-oxygen-voltage (LOV) domain of Phaeodactylum tricornutum Aureochrome 1a $(\mathrm{AuLOV})$ is a conformational-driven allosteric protein. ${ }^{32}$ AuLOV is a monomer in the dark state and undergoes dimerization upon blue light perturbation. ${ }^{33}$ In both cases, the ensembled model ranked the allosteric sites top 1 with probabilities of $45.14 \%$ and $89.46 \%$, respectively. This indicates that the ensembled model is capable of predicting both dynamicsdriven and conformational-driven allosteric proteins. 
(A)

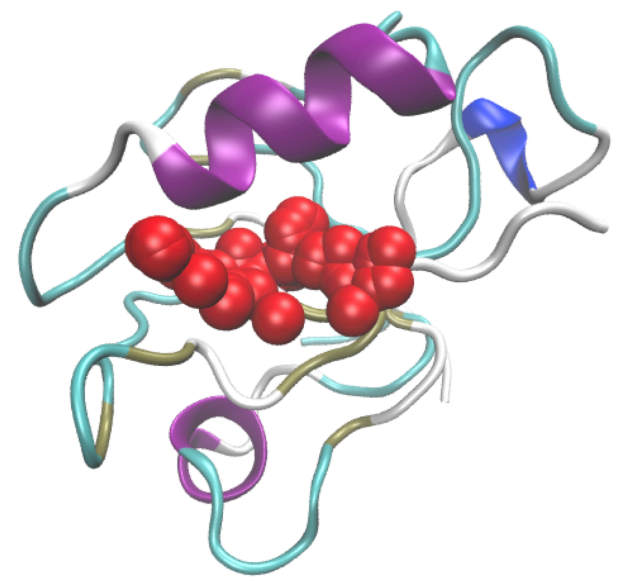

(B)

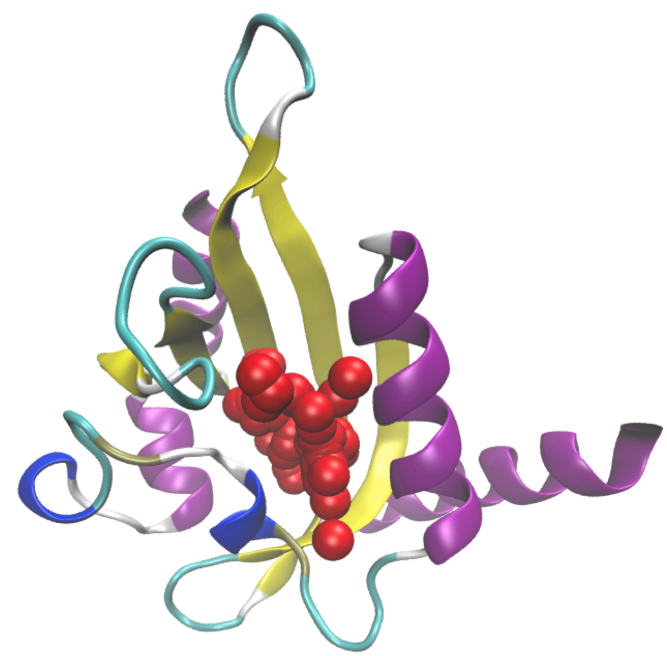

Figure 7: Two examples of predicted results. (A) Dynamics-driven PDZ2 protein in bound states (PDB ID 3LNY). (B) The LOV domain of conformational-driven Phaeodactylum tricornutum Aureochrome 1a in dark state (PDB ID: 5DKK). Red regions are the most probable pockets in the predicted results with probabilities of (A) $45.14 \%$ and (B) $89.46 \%$ and are also the true allosteric sites.

\subsection{Web and CLI Usage}

The web server was implemented using a Python web framework Django. JSmol is a JavaScript implementation of the Jmol package and was embedded in the web page for protein and pocket visualization. Web page was rendered using Bootstrap. A workflow is outlined in Figure 8.

An example of input and output of PASSer is displayed in Figure 9. User can submit a PDB ID if available or upload a custom PDB file as shown in Figure 9A. By default, all chains in the protein are analyzed. Prediction results are split into two parts (Figure 9B and C). Top 3 pockets with the highest probability are summarized in a table. For each pocket, the corresponding residues can be retrieved by clicking the "Show Residues" texts. Protein structure is visualized using JSmol. Three pockets are shown upon user clicking "Load pocket" icon and hidden by clicking "Hide pocket" or "Reset" icons.

A command line interface is provided to facilitate potential developments. Similar with the web usage, this CLI can take either a PDB ID or a local PDB file for prediction. 


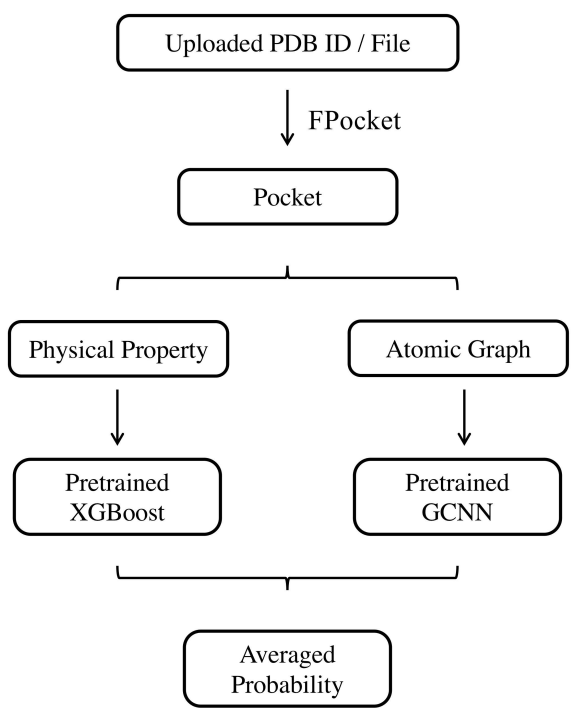

Figure 8: Workflow of web server. User can upload either a PDB ID or a PDB file. FPocket is used to detect pockets. For each pocket, physical properties are calculated and predicted using pretrained XGBoost model; while an atomic graph is constructed and fed into pretrained GCNN model. A final probability is given by averaging results from both models.

\section{Discussion}

The dataset quality for model training is of foundamental importance. Classification models tend to fail in prediction performance and lack of generalization with poor-collected dataset, such as not enough training data and high similarity between structures. The powerful ASD is an online database that provides allosteric proteins and sites with high resolution, which brings new opportunity for allosteric site prediction. There are other database like ASBench ${ }^{34}$ and sc-PDB ${ }^{35}$ that can also be used for to improve the data quality and model performance.

In order to predict allosteric sites, proper pockets need to be detected on the surface of proteins. Several open-source pocket detection softwares have been developed. Previous results $^{27}$ have shown that, the geometry-based detection algorithm FPocket is superior compared with other methods, such as PASS ${ }^{36}$ and LIGSITE ${ }^{\csc 37}$ and can cover known allosteric 
(A)

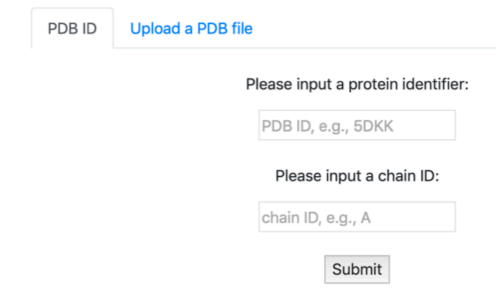

(B)

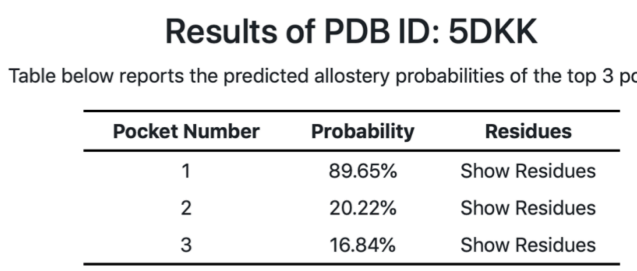

(C)

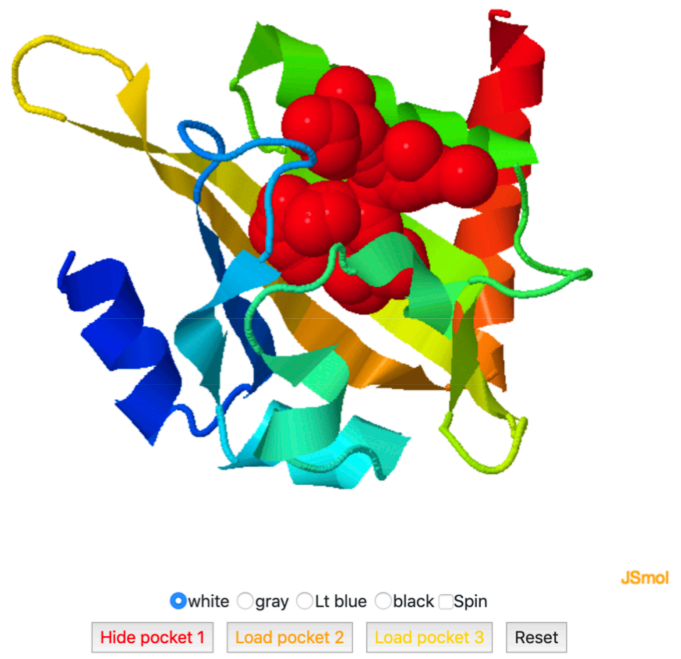

Figure 9: Web interface and usage. (A) User can either submit a PDB ID or a PDB file. (B) Predicted results are summarized in a table with corresponding probabilities and pocket residues. (C) Protein structures and pocket sites are visualized in an interactive window.

sites. Moreover, FPocket is actively developed and can be integrated with other methods to build a complete pipline for sites prediction.

Several computational methods have been developed for allosteric site prediction over the past years. Especially, due to the fast development of machine learning (ML), many models integrate ML for accurate prediction, ${ }^{11,17}$ such as support vector machine and random forest. One critical issue is that, many ML models fail when dealing with imbalanced dataset $^{38}$ : in the allosteric site database, negative samples account for a majority of the dataset with small portion of positive samples. Undersampling is one solution to rebalance dataset. For example, Allosite discarded some negative labels and used a ratio of 1:4 between positive and negative labels. However, undersampling leads to insufficient usage of the overall dataset. A recently developed gradient boosing method, XGBoost, overcomes this data imbalance by controling relative weights between classes so that the database can be fully utilized. Various performance indicators, as shown in Table 4, validated the effectiveness of XGBoost in the identification of allosteric sites.

It is worth noting that some features are highly correlated, as shown in Figure 4. This 
collinearity should be addressed in regression models, which reduces the model precision and thus weakens prediction results. In contrast, XGBoost is immune to this problem. When selecting features that are highly correlated, XGBoost will choose only one of them. Therefore, collinearity does not affect the prediction results. Nevertheless, collinearity influences model interpretation, e.g., feature importance, which should be addressed with caution.

Physical properties have been widely used to describe pocket features. These features are normally calculated using the static structure of protein. To prob the dynamical behavior of pockets, normal mode analysis and MD simulations are normally conducted. ${ }^{7,39}$ Results from these methods have shown that models can achieve satisfatory performance through the combination of both static features and protein dynamics. Nevertheless, the geometry is often ignored, which could play an important role in pocket prediction. Graph convolutional neural network was applied to study the topology information. Specifically, pockets were treated as undirected graphs and GCNN was designed to learn the local connectivity among atoms. While a previous study ${ }^{9}$ included energy-weighted covalent and week bonds in the prediction of allosteric sites, it should be noted here that : (1) it is believed the physical properties are covered in the site descriptors so GCNN only study the node degree; (2) GCNN does not require any prior information about the location of active site. The ensembled model, consisting of GCNN and XGBoost, exihibited good performance compared with single models.

\section{Conclusion}

The proposed ensembled model is composed of XGBoost and GCNN that can learn both physical properties and topology of protein pockets. The results are comparable with previous studies and have higher percentage in ranking allosteric sites at top positions. The web server provides user-friendly interface. Protein structures and top pockets are visualized in an interactive window on the result page. This ensembled model, embedded in the PASSer 
and CLI, can help exploration on protein allostery and drug development.

\section{Data availability}

The authors declare that all data supporting the findings of this study are available within the paper and its Supplementary Information files.

\section{Code availability}

The PASSer server is available at https://passer . smu . edu. The command line interface is available on GitHub at https://github.com/smutaogroup/passerCLI.

\section{References}

(1) Hilser, V. J. An ensemble view of allostery. Science 2010, 327, 653-654.

(2) Nussinov, R.; Tsai, C.-J.; Csermely, P. Allo-network drugs: harnessing allostery in cellular networks. Trends in pharmacological sciences 2011, 32, 686-693.

(3) Pei, J.; Yin, N.; Ma, X.; Lai, L. Systems biology brings new dimensions for structurebased drug design. Journal of the American Chemical Society 2014, 136, 11556-11565.

(4) Wenthur, C. J.; Gentry, P. R.; Mathews, T. P.; Lindsley, C. W. Drugs for allosteric sites on receptors. Annual review of pharmacology and toxicology 2014, 54, 165-184.

(5) Motlagh, H. N.; Wrabl, J. O.; Li, J.; Hilser, V. J. The ensemble nature of allostery. Nature 2014, 508, 331-339.

(6) Laine, E.; Goncalves, C.; Karst, J. C.; Lesnard, A.; Rault, S.; Tang, W.-J.; Malliavin, T. E.; Ladant, D.; Blondel, A. Use of allostery to identify inhibitors of calmodulin- 
induced activation of Bacillus anthracis edema factor. Proceedings of the National Academy of Sciences 2010, 10\%, 11277-11282.

(7) Panjkovich, A.; Daura, X. Exploiting protein flexibility to predict the location of allosteric sites. BMC bioinformatics 2012, 13, 273.

(8) Qi, Y.; Wang, Q.; Tang, B.; Lai, L. Identifying allosteric binding sites in proteins with a two-state Go model for novel allosteric effector discovery. Journal of chemical theory and computation 2012, 8, 2962-2971.

(9) Amor, B. R.; Schaub, M. T.; Yaliraki, S. N.; Barahona, M. Prediction of allosteric sites and mediating interactions through bond-to-bond propensities. Nature communications 2016, $7,1-13$.

(10) Bian, Y.; Jing, Y.; Wang, L.; Ma, S.; Jun, J. J.; Xie, X.-Q. Prediction of orthosteric and allosteric regulations on cannabinoid receptors using supervised machine learning classifiers. Molecular pharmaceutics 2019, 16, 2605-2615.

(11) Huang, W.; Lu, S.; Huang, Z.; Liu, X.; Mou, L.; Luo, Y.; Zhao, Y.; Liu, Y.; Chen, Z.; Hou, T., et al. Allosite: a method for predicting allosteric sites. Bioinformatics 2013, 29, 2357-2359.

(12) Song, K.; Liu, X.; Huang, W.; Lu, S.; Shen, Q.; Zhang, L.; Zhang, J. Improved method for the identification and validation of allosteric sites. Journal of Chemical Information and Modeling 2017, 57, 2358-2363.

(13) Greener, J. G.; Sternberg, M. J. AlloPred: prediction of allosteric pockets on proteins using normal mode perturbation analysis. BMC bioinformatics 2015, 16, 1-7.

(14) Goncearenco, A.; Mitternacht, S.; Yong, T.; Eisenhaber, B.; Eisenhaber, F.; Berezovsky, I. N. SPACER: server for predicting allosteric communication and effects of regulation. Nucleic acids research 2013, 41, W266-W272. 
(15) Panjkovich, A.; Daura, X. PARS: a web server for the prediction of protein allosteric and regulatory sites. Bioinformatics 2014, 30, 1314-1315.

(16) Suykens, J. A.; Vandewalle, J. Least squares support vector machine classifiers. Neural processing letters 1999, 9, 293-300.

(17) Chen, A. S.-Y.; Westwood, N. J.; Brear, P.; Rogers, G. W.; Mavridis, L.; Mitchell, J. B. A random forest model for predicting allosteric and functional sites on proteins. Molecular informatics 2016, 35, 125-135.

(18) Liaw, A.; Wiener, M., et al. Classification and regression by randomForest. $R$ news 2002, 2, 18-22.

(19) Chen, T.; Guestrin, C. Xgboost: A scalable tree boosting system. Proceedings of the 22nd acm sigkdd international conference on knowledge discovery and data mining. 2016; pp 785-794.

(20) Basit, A. H.; Abbasi, W. A.; Asif, A.; Gull, S.; Minhas, F. U. A. A. Training hostpathogen protein-protein interaction predictors. Journal of bioinformatics and computational biology 2018, 16, 1850014.

(21) Li, K.; Zhang, S.; Yan, D.; Bin, Y.; Xia, J. Prediction of hot spots in protein-DNA binding interfaces based on supervised isometric feature mapping and extreme gradient boosting. BMC bioinformatics 2020, 21, 1-10.

(22) Kipf, T. N.; Welling, M. Semi-supervised classification with graph convolutional networks. arXiv preprint arXiv:1609.02907 2016,

(23) Coley, C. W.; Jin, W.; Rogers, L.; Jamison, T. F.; Jaakkola, T. S.; Green, W. H.; Barzilay, R.; Jensen, K. F. A graph-convolutional neural network model for the prediction of chemical reactivity. Chemical science 2019, 10, 370-377. 
(24) Ryu, S.; Kwon, Y.; Kim, W. Y. A Bayesian graph convolutional network for reliable prediction of molecular properties with uncertainty quantification. Chemical Science 2019, 10, 8438-8446.

(25) Torng, W.; Altman, R. B. Graph convolutional neural networks for predicting drugtarget interactions. Journal of Chemical Information and Modeling 2019, 59, 41314149 .

(26) Huang, Z.; Zhu, L.; Cao, Y.; Wu, G.; Liu, X.; Chen, Y.; Wang, Q.; Shi, T.; Zhao, Y.; Wang, Y., et al. ASD: a comprehensive database of allosteric proteins and modulators. Nucleic acids research 2011, 39, D663-D669.

(27) Le Guilloux, V.; Schmidtke, P.; Tuffery, P. Fpocket: an open source platform for ligand pocket detection. BMC bioinformatics 2009, 10, 1-11.

(28) Pedregosa, F.; Varoquaux, G.; Gramfort, A.; Michel, V.; Thirion, B.; Grisel, O.; Blondel, M.; Prettenhofer, P.; Weiss, R.; Dubourg, V., et al. Scikit-learn: Machine learning in Python. the Journal of machine Learning research 2011, 12, 2825-2830.

(29) Xu, K.; Hu, W.; Leskovec, J.; Jegelka, S. How powerful are graph neural networks? arXiv preprint arXiv:1810.00826 2018,

(30) Wang, M.; Yu, L.; Zheng, D.; Gan, Q.; Gai, Y.; Ye, Z.; Li, M.; Zhou, J.; Huang, Q.; Ma, C., et al. Deep graph library: Towards efficient and scalable deep learning on graphs. arXiv preprint arXiv:1909.01315 2019,

(31) Zhou, H.; Dong, Z.; Tao, P. Recognition of protein allosteric states and residues: Machine learning approaches. Journal of Computational Chemistry 2018, 39, 1481-1490.

(32) Heintz, U.; Schlichting, I. Blue light-induced LOV domain dimerization enhances the affinity of Aureochrome 1a for its target DNA sequence. Elife 2016, 5, e11860. 
(33) Tian, H.; Trozzi, F.; Zoltowski, B. D.; Tao, P. Deciphering the Allosteric Process of Phaeodactylum tricornutum Aureochrome 1a LOV Domain. The Journal of Physical Chemistry B 2020,

(34) Huang, W.; Wang, G.; Shen, Q.; Liu, X.; Lu, S.; Geng, L.; Huang, Z.; Zhang, J. ASBench: benchmarking sets for allosteric discovery. Bioinformatics 2015, 31, 25982600.

(35) Desaphy, J.; Bret, G.; Rognan, D.; Kellenberger, E. sc-PDB: a 3D-database of ligandable binding sites - 10 years on. Nucleic acids research 2015, 43, D399-D404.

(36) Brady, G. P.; Stouten, P. F. Fast prediction and visualization of protein binding pockets with PASS. Journal of computer-aided molecular design 2000, 14, 383-401.

(37) Huang, B.; Schroeder, M. LIGSITE csc: predicting ligand binding sites using the Connolly surface and degree of conservation. BMC structural biology 2006, 6, 19.

(38) Zhao, X.-M.; Li, X.; Chen, L.; Aihara, K. Protein classification with imbalanced data. Proteins: Structure, function, and bioinformatics 2008, 70, 1125-1132.

(39) Penkler, D.; Sensoy, O.; Atilgan, C.; Tastan Bishop, O. Perturbation-response scanning reveals key residues for allosteric control in Hsp70. Journal of Chemical Information and Modeling 2017, 5\%, 1359-1374.

\section{Acknowledgement}

Computational time was generously provided by Southern Methodist University's Center for Research Computing. Research reported in this paper was supported by the National Institute of General Medical Sciences of the National Institutes of Health under Award No. R15GM122013. 


\section{Competing interests}

The authors declare no competing interests 